\title{
Effect of pesticides on fetal development in the intrauterine and early postnatal periods
}

\author{
Anastasia Derevtsova ${ }^{1, *}$, Andrey Kaviev ${ }^{1}$, Sardorbek Makhkamov ${ }^{1}$, Bakhtiyor \\ Abdulazizov $^{1}$, and Alexandra Shevtsova ${ }^{1}$ \\ ${ }^{1}$ Far Eastern Federal University, 8, Sukhanova, Vladivostok, 690950, Russia
}

\begin{abstract}
The main objective of this study was to determine the level of toxic effects of pesticides on the development of immune and endocrine systems in offspring during intrauterine and early postnatal development. During the experiment, preparations containing the pesticide lambdaCyhalothring (LCP) and fipronil were injected into white laboratory rats during pregnancy and lactation. The study was carried out using electron microscopy and morphological and biochemical studies. From the results of this study, it is concluded that the toxic effect of pesticide exposure during pregnancy and lactation is manifested by stunted growth of the thyroid gland (lymph nodes), thymus, lymph node and spleen development. Cell proliferation and apoptosis caused by direct toxic effects of drugs, developed hypothyroidism, oxidative stress reactions in both mother-nature and offspring are the main cause of toxic effects of industrial pesticides on the immune and endocrine systems. It follows from the data obtained that early detection of hypothyroidism and oxidative stress states in pregnant women and infants and then their correction with drugs is an important aspect of public health.
\end{abstract}

\section{Introduction}

The endocrine and immune systems, along with the nervous system, are extremely sensitive to the action of most environmental chemical pollutants. The terms "immunotoxicity" (IT, immunotoxicity) and "endocrine-disrupting chemicals" (ED, endocrine-disrupting chemicals or endocrine disruptors) have been firmly established in the literature for over three decades [1]. Endocrine-disrupting chemicals adversely affect various steps of hormone synthesis, transport, and function, and can also act as agonists or antagonists of natural hormones and cause a certain hormonal imbalance in the body. The term "immunotoxicity" refers not only to the direct toxic effects of various environmental pollutants on immune cells, but also to disturbances in the regulation of immunogenesis and the body's immune reactions, including immunosuppression, allergic reactions, autoimmune diseases and other immunopathological conditions [2, 4]. The predominant part of environmental pollutants are pesticides, without the use of which the prospective development of agriculture is impossible. It has now been established that a number of pesticides, including the latest generations, have immunotoxic and endocrine-disrupting

* Corresponding author: Klarunia1998@yandex.ru 
properties. However, the vast majority of studies on the mechanisms of immune and endocrine toxicity of pesticides have been performed in adult humans and sexually mature animals, or under in vitro conditions. Meanwhile, the immune and endocrine system of the fetus and neonates is incomparably more sensitive to the action of various toxic substances compared to adults $[4,5]$. In recent years, there has been increasing evidence that exposure to pesticides in utero or early childhood leads to a significant increase in chronic diseases in children, such as asthma, autoimmune, infectious and other diseases, including cancer [1, $2]$.

Unfortunately, the problem of "developmental immunotoxicity" (developmental immunotoxicity), that is, the mechanisms of adverse effects of pesticides on the developing immune system of offspring, is still far from being finally solved. The available studies are fragmentary and do not provide a complete picture of the mechanisms of toxic effects of pesticides on new-borns and children when they enter the body through the mother. Undoubtedly, revealing the mechanisms of immunotoxic and endocrine-disrupting effects of pesticides will contribute to the development of methods of secondary prevention and pathogenetic therapy of toxic effects of pesticides on the younger generation in conditions of intoxication through the maternal organism [3, 4].

Purpose of work: to reveal structural and functional mechanisms of pesticide toxic effects on postnatal development of immune and endocrine systems of offspring under conditions of their exposure through maternal organism.

\section{Materials and methods}

The pyrethroid insecticide lambda-cyhalothrin (LCT) and the benzopyrazole pesticide fipronil (FPN), widely used in our country and all over the world, were selected for experiments.

Lambda-cyhalothrin was created in 1977 as a powerful $\alpha$-cyano-SP type II insecticide. It is a nerve poison that acts by disrupting the ionic conductivity of nerve membranes by prolonging the $\mathrm{Na}+$ current, which leads to paralysis and death of parasites [5]. It has effective, fast and persistent action against various arthropods that affect human and animal health. It is highly recommended in vector control of infectious diseases, in veterinary and agricultural products to control a variety of insects on vegetables, fruits, cotton and field crops [6]. 


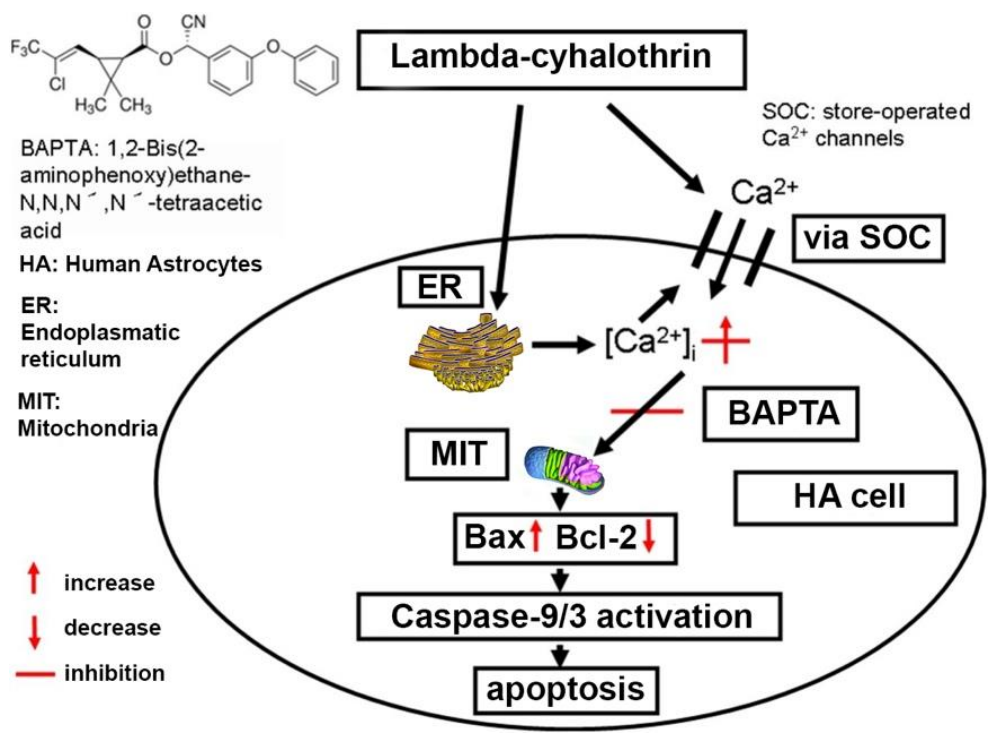

Fig. 1. Effect of lambda-cyhalothrin on $\mathrm{Ca} 2+$ homeostasis and activated $\mathrm{Ca} 2+-$ associated mitochondrial apoptotic pathway in normal astrocytes.

Lambda-cyhalothrin is stable and not washed off by rain as it quickly passes through the leaf cuticle (after 1 hour). It is stable in light. Half-life in soil $4-12$ weeks. Half-life in apples and cabbage is 2-5 days. Low toxicity to birds, no harm to earthworms. Toxic to bees and other beneficial insects. Highly toxic to fish. Does not irritate skin, weakly irritate eyes. Clinical picture of acute lambda-cyhalothrin poisoning represents clinical signs of pyrethroid intoxication (effects on central and peripheral nervous system): discoordination of movements, salivation, tremor, respiratory disorders, reduced muscle tone. Drugs based on lambda-cyhalothrin are class 2 and class 3 for humans $[5,6]$

Fipronil is a widely used phenylpyrazole insecticide with systemic action. Fipronil acts as a non-competitive blocker of gamma-aminobutyric acid (GABA)-controlled chloride channels in the central nervous system, ultimately leading to insect mortality. Fipronil exhibits selective toxicity to insect GABA receptors and is therefore often used as an alternative to other pesticides in many conditions, including agricultural, commercial and industrial applications. In addition, target insects have no known resistance to pesticides, a phenomenon that has been shown to occur for most other pesticides. This, in turn, has led to a rapid increase in the use of fipronil, which accounts for approximately $10 \%$ of the global pesticide market. However, the toxic properties of fipronil persist relatively long in aerobic soils and water, with half-lives ranging from 2 days to 7.3 months, depending on substrate and conditions $[7,8]$. 


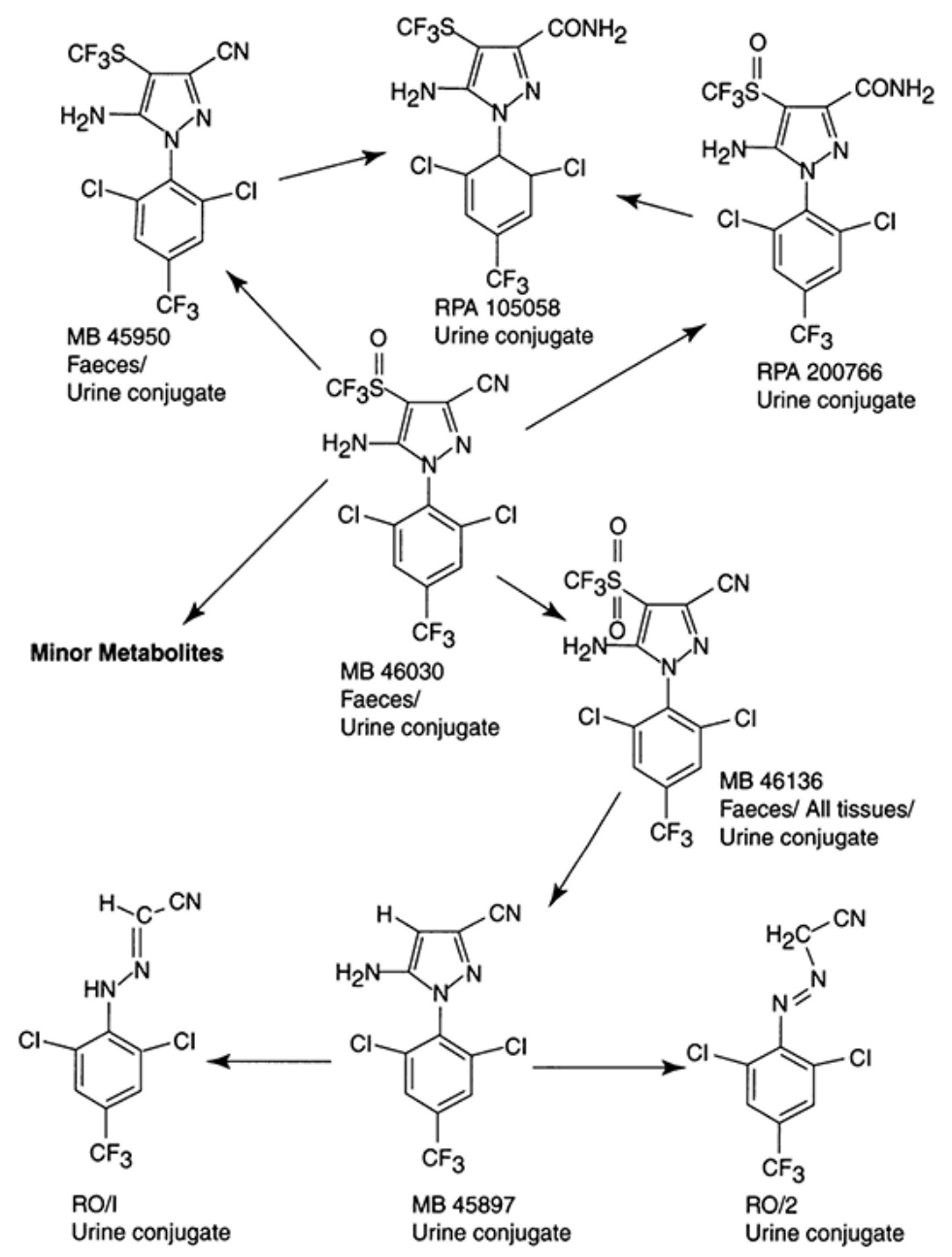

Fig. 2. Proposed metabolie pathway for fipronil in the rat. (From ACP 1999.)

Experiments were performed on white mature non-pregnant female rats weighing 150$180 \mathrm{~g}$, mature male rats were used only for fertilization. Female rats were divided into three groups of 45 rats each. The first group of rats was injected by mouth using a probe diluted in physiological solution with LCT at the rate of $8 \mathrm{mg} / \mathrm{kg} /$ day. The second group of rats received FPN at the rate of $3.6 \mathrm{mg} / \mathrm{kg} /$ day in the same way. The dose of both drugs was $1 / 100$ of the drug's LD50. The third group (control) received an equal volume of sterile saline. The administration of both pesticides to the experimental groups of rats was performed daily for 75 days until the end of the experiments. On day 31 of the experiments, females of all groups were mated with males for fertilization. The onset of pregnancy was monitored by the presence of spermatozoa in vaginal smears. After the onset of pregnancy, females were separated from males and placed in separate cages for further studies [8,9].

Offspring from all groups of animals were selected one, two, three weeks and one month after birth, in the morning hours, on an empty stomach, under light ether anesthesia. Thymus, mesenteric lymph nodes, spleen and thyroid gland were studied by morphometric, ultrastructural and immunohistochemical methods. Besides, the concentration of thyroid and thyroid hormones was determined, and the amount of free radicals of oxidative stress in 
blood serum of all animals was determined [9, 10]. All numerical data were statistically processed using a package of computer programs, differences satisfying $\mathrm{P}<0.05$ were considered reliable.

\section{Results}

Exposure of the offspring to pesticides through the maternal body resulted in immunotoxic effect, which manifested itself in the form of impaired postnatal growth and formation of the organs of the immune system. The formation of structurally functional T- and Bdependent zones of mesenteric lymph nodes and spleen in experimental rats was significantly delayed, being carried out 7-10 days later compared to the control. Moreover, the delay in the rate of formation of mesenteric lymph node zones was more pronounced under FPN intoxication compared with LST exposure. Electron microscopic studies revealed high functional activity of macrophages and destructive changes in subcellular organelles of lymphoid cells. These changes were predominantly observed in the Tdependent zones (paracortical zone of mesenteric lymph nodes and periarteriolar zone of the white pulp of the spleen) of the organs and occurred significantly more frequently when exposed to FPN. Morphometric studies also showed a significant decrease in the growth rate and establishment of lymphoid tissue of the organs in offspring obtained under exposure to pesticides through the maternal body. Immunohistochemical studies showed that pesticide exposure leads to inhibition of proliferative activity of lymphoid cells of mesenteric lymph nodes and spleen, especially in the T-dependent zones of the organs. When exposed to LST, the proliferative index of cells in these zones was $10-15 \%$, and when exposed to FPN, it was $15-30 \%$ lower than that of controls. At the same time, intrauterine and early postnatal exposure to pesticides resulted in a significant increase in the degree of cell apoptosis. Apoptotic cells were most frequent in T-dependent zones compared to lymphatic follicles (B-dependent zones). The greatest increase in the apoptosis index was observed on 14-21 days after birth, when the indexes of experimental animals were 3.2-4.3 times higher than those of control animals. At the same time, the induction of cell apoptosis was manifested to a significantly higher degree when exposed to FPN than when intoxicated with LST [10, 11,12].

The immunotoxic effect of pesticides was most pronounced in the thymus gland. The growth rate of the average thymus lobule area under the influence of LST by $10-15 \%$, and under FPN application by 15-30\% lagged behind the control parameters. The area occupied by the cortical area of the thymus decreased by $15-25 \%$ and $20-40 \%$ respectively.

Along with this, certain disorders of postnatal growth and development of thymus microenvironment cells were revealed. Starting from the moment of birth, in thymus of experimental animals there was a tendency to decrease the number of epitheliooreticular cells (ERC) per unit area of the lobule. Moreover, the average number of ERK decreased more clearly in the cortical zone of thymus. Electron microscopy revealed hypoplasia of endoplasmic network and Golgi complex as well as heterogeneity of secretory vacuoles in ERK of experimental animals, indicating impaired secretory activity of cells. A large number of heterophagosomes with thymocyte residues were detected in their cytoplasm. Exposure to pesticides led to inhibition of proliferative activity of lymphoid cells of thymus (thymocytes), especially in the cortical zone of the organ. When exposed to LST, the proliferative index of cells in these zones was $35-40 \%$, and when exposed to FPN, it was $45-55 \%$ lower than that of control. At the same time, immunotoxic effects of pesticides contributed to a significant increase in thymocyte apoptosis. Cells subjected to apoptosis were most frequent in the cortical zone of the thymus. Quantitative counting showed that under LST exposure the thymocyte apoptosis index in this zone averaged $40-45 \%$, and under FPN exposure it was 50-55\% higher than that of the control group [13, 14,15$]$. 


\section{Discussion}

To a greater extent, the thyroid functions in female rats and their offspring were affected by pesticides. However, given the high level of thyroid hormone, the concentration of free thyroxine (T4), triiodothyronine (T3) was much lower compared with the control group. Molecular morphometry showed that the growth rate of the total follicular epithelial area under LCT exposure was 10-17\%, while it lagged behind the controls by an average of 15$30 \%$ under FPN exposure. Based on this study, it was found that exposure to pesticides leads to a decrease in the follicular epithelial area and the follicular epithelium of the thyroid gland as a whole. Reduced functional activity of the thyroid gland, in turn, led to retardation of its growth and formation. The most pronounced hypothyroidism was observed in LCT-exposed progeny compared with FPN-exposed progeny. Electron microscopy showed a decrease in the size of mitochondria in the cells as well as in the endoplasmic reticulum and thyrocytic Golgi complexes, indicating decreased secretory function of the cells. Pesticide exposure caused inhibition of thyroid cell proliferation. At the same time, intrauterine and early infantile exposure of the thyroid gland to chemicals led to a marked increase in the degree of apoptosis of its cells. The offspring apoptosis index after exposure of the thyroid gland to FPN was 4.5 times higher compared to controls and almost 3.5 times higher compared to LCT exposure.

The results of this study suggest that the toxic effects of pesticides on the development of the endocrine and immune systems are associated with a number of metabolic changes in organs or tissues. The results of this study suggest that the toxic effects of pesticides on the development of the endocrine and immune systems are associated with a number of metabolic changes in organs or tissues. The studies we studied showed the endocrine, or rather thyroid-damaging effects of pesticides, which were the likely cause of hypothyroidism in the mother's womb and offspring. In addition, we found an important role of oxidative stress - the main inducer of cellular apoptosis $[11 ; 12]$. Thus, it can be concluded that the induction of apoptosis in the offspring is due not only and not so much to the direct toxic effect of pesticides. The induction of apoptosis is mainly mediated by the weakening of the regulatory role of thyroid hormones on the processes of cell proliferation and apoptosis due to hypothyroidism, as well as an increase in the amount of free radicals as a result of oxidative stress.

\section{Conclusion}

1. The influence of even relatively small doses of pesticides during pregnancy and breastfeeding leads to the development of immunotoxic and endocrine-destroying effects in offspring; at the same time, the pesticide "Fiproniel" has a more pronounced toxic effect compared to lambda-cyhalothrin.

2. As a result of the immunotoxic effect of pesticides, delayed postnatal growth and the formation of peripheral organs of the immune system (lymph nodes and spleen), violations of the secretory function of the thymus and the formation of thymus-dependent zones by the mesentery and spleen lymph nodes occur.

3. Endocrine disorder is caused by the predominance of disorders of the structure and function of the thyroid gland in the mother's body, leading to the development of hypothyroidism in the offspring.

4. In the pathogenesis of immunotoxic and endocrine-destroying effects the leading role is played by hypothyroidism and oxidative stress that contribute to the induction of apoptosis while suppressing the proliferation of immune-endocrine system cells. 


\section{References}

1. M.A. La Merrill et al., Nature Reviews Endocrinology 16.1, 45-47 (2020)

2. A. Jabłońska-Trypuć et al., Journal of Environmental Science and Health B.52.7, 483393 (2017)

3. Young Ah Kim et al., International journal of hygiene and environmental health 222.3, 524-532 (2019)

4. S.R. Khalil, et al., Fish \& shellfish immunology 100, 208-218 (2020)

5. Akash Sabarwal, Kunal Kumar, R.P. Singh, Environmental toxicology and pharmacology 63, 103-114 (2018)

6. Hsu Shu-Shong, Chung-Ren Jan, Wei-Zhe Liang, Neurotoxicology 69, 97-107 (2018)

7. E.V.S. Hessel et al., Critical reviews in toxicology 45.1, 68-82 (2015)

8. J-M. Bonmatin et al., Environmental Science and Pollution Research 22.1, 35-67 (2015)

9. Xianjiang Li et al., Trends in Food Science \& Technology 97, 185-195 (2020)

10. Cyril Vidau et al., Toxicology in Vitro 23.4, 589-597 (2009)

11. José M. Matés et al., Archives of toxicology 86.11, 1649-1665 (2012)

12. M.A. Robea et al., Survey in Fisheries Sciences 5.1, 20-31 (2018)

13. Gun-Hwi Lee, Choi Kyung-Chul, Comparative Biochemistry and Physiology Part C: Toxicology \& Pharmacolog 235, 108789 (2020)

14. T. Fukuyama, T.-N. Risako, Pesticide and Immunotoxicology, Allergy and Immunotoxicology in Occupational Health-The Next Step (Springer, Singapore, 2020)

15. R. Lauretta, et al., Frontiers in endocrinology 10, 178 (2019) 